\title{
Mit MINERVA am Stau vorbei - On-Board-Verkehrsinformation im städtischen Wirtschaftsverkehr
}

\author{
Bertram Meimbresse, Christine Behnke, Herbert Sonntag
}

Zusammenfassung

Kraftfahrer in städtischen Gebieten haben oft nur beschränkte Kenntnisse über die aktuelle Verkehrslage, in den Verkehrsmanagementzentralen sind jedoch umfangreiche Verkehrsinformationen vorhanden. Im Forschungsprojekt MINERVA wurde ein System entwickelt, welches Kraftfahrern automatisch relevante Verkehrsinformationen auf ein mobiles Endgerät zur Verfügung stellt, um so deren intuitives Routing zu optimieren. Die Ergebnisse zeigen, dass On-Board-Verkehrsinformationen das intuitive Routing verbessern können.

\section{Abstract}

Drivers in urban areas are mostly not sufficiently informed about the present traffic situation, but comprehensive traffic information is available in traffic management offices. Within the research project MINERVA a system for the automatic supply of relevant up-to-date traffic information on a mobile device for the driver is developed to improve their intuitive routing. The comparison shows, that on-board-trafficinformation can improve the intuitive routing.

\section{Straßen-Wirtschaftsverkehr in Ballungsgebieten}

Der städtische Straßen-Wirtschaftsverkehr hat wichtige Funktionen für die Versorgung der Bevölkerung sowie für den Transport von Gütern für die ansässige Industrie. Die Definition des Wirtschaftsverkehrs reicht dabei vom »reinen« Güterverkehr über Verkehre zu Ver- und Entsorgungszwecken, Liefer- und Geschäftsfahrten bis zum privaten Einkaufsverkehr (u. a. Dasen et al. 2001; Wermuth et al. 1998). Hier soll weiterhin eine Definition des Straßen-Wirtschaftsverkehrs verwendet werden, welche mittlerweile häufig herangezogen wird: Straßen-Wirtschaftsverkehr sind alle Fahrten mit Kraftfahrzeugen in Ausübung des Berufes, welche nicht der Ortsveränderung vom Wohnort zur Arbeitsstelle und zurück dienen.

Auf den Straßen-Wirtschaftsverkehr und die Einhaltung von Lieferzeitfenstern hat die Verkehrssituation großen Einfluss. Vor allem in städtischen Gebieten ist die aktuelle Verkehrssituation auf Grund unvorhersehbarer Verkehrsstörungen jedoch schwer vorherzusagen.

\section{Routenberechnung}

\subsection{Funktionsweise der Routenberechnung}

Die Routenberechnung ist ein Optimierungsproblem, bei dem der kürzeste Weg zwischen zwei oder mehr Punkten bei positiver Kantenbewertung errechnet werden soll. Das Straßennetz wird als Netzwerkgraph betrachtet, bestehend aus Knoten (Orte, an denen eine Entscheidung getroffen werden muss, zum Beispiel Autobahnabfahrten oder Straßenkreuzungen) und Kanten (Straßenverbindungen zwischen zwei Knoten).

Bei der Routenberechnung stehen in der Regel zwei Optimierungskriterien zur Auswahl: schnellste und kürzeste Route. Bei der Berechnung der schnellsten Route (Minimierung der Fahrzeit) werden Geschwindigkeiten oder Fahrzeiten als Kantenbewertung verwendet. Zur Berechnung der kürzesten Route (Minimierung der Entfernung) wird die Länge des Weges als Kantenbewertung genutzt.

Bei der Routenberechnung in handelsüblichen Navigationssystemen werden nur für Anfang und Ende des Weges alle Straßentypen einbezogen; für den Hauptteil des Weges wird lediglich das übergeordnete Straßennetz zugrunde gelegt. 
Für die Routenberechnung wird oft eine Kombination der Algorithmen Dijkstra und $A^{*}$ angewendet. In einem Netzwerkgraphen (siehe Abbildung 1) lässt sich für einen gegebenen Startknoten $s$ für jeden von $s$ erreichbaren Knoten $z$ der kürzeste Pfad mit dem Dijkstra-Algorithmus bestimmen, wenn die Kantenbewertung nichtnegativ ist. Die Distanz vom aktuellen Knoten $i$ zu $s$ wird bestimmt aus der Distanz des Vorgängers $i-1$ zu s, addiert mit der Kantenlänge zwischen dem aktuellen Knoten $i$ und dem Vorgänger-Knoten $i$-1. Die jeweils kürzeste Distanz von $s$ zum aktuellen Knoten $i$ wird gespeichert, längere Distanzen werden überschrieben und verfallen. Es wird jeweils der Knoten betrachtet, welcher die kürzeste Distanz zu $s$ aufweist und noch nicht abschließend betrachtet wurde. Der Algorithmus hat den kürzesten Weg von $s$ nach $z$ gefunden, wenn alle Distanzen zu anderen Knoten länger als die Distanz zu z sind oder wenn alle anderen Knoten abschließend betrachtet wurden.

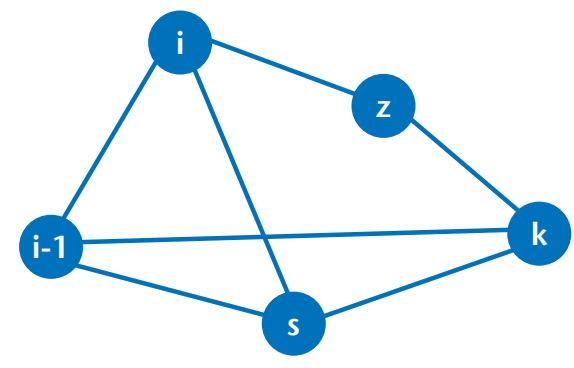

Abb. 1: Netzwerkgraph

Der Dijkstra-Algorithmus (Dijkstra 1959) bietet eine optimale jedoch zeitlich aufwendige Lösung. Da Straßennetze in der Regel sehr umfangreich sind (zum Beispiel mehr als 42 Millionen gerichtete Kanten im westeuropäischen Straßennetz), ist der Dijkstra-Algorithmus nur bedingt geeignet (Schultes 2008). Um die Laufzeit der Routenberechnung zu verkürzen, kann mit dem A* Algorithmus (Hart 1968) zielgerichtet gesucht werden: Es wird eine Abschätzung des verbleibenden Routingpfades vorgenommen und zur Auswahl des nächsten, vielversprechendsten Berechnungsschrittes genutzt. Es gibt noch weitere Ansätze, um die Routenberechnung $\mathrm{zu}$ vereinfachen und $\mathrm{zu}$ beschleunigen, zum Beispiel das Highway-Routing (Schultes 2008).

\subsection{Routingansätze}

Unter Routing sollen hier die Ergebnisse von Routenberechnungen mit unterschiedlichen Randbedingungen verstanden werden bzw. der von Fahrern aufgrund ihrer Kenntnisse gewählte Weg als Abfolge von Kanten im Netz.

\section{Statisches Routing}

Die Berechnung für das Statische Routing wird mit Hilfe eines Straßennetzes mit festen Kantenbewertungen durchgeführt. Das Ergebnis der Routenberechnung zwischen zwei Punkten ist unabhängig vom Zeitpunkt der Fahrt gültig. Sinnvoll ist das Statische Routing vor allem, wenn die Entfernung minimiert werden soll, oder in Gebieten, in denen sehr wenige Verkehrsstörungen auftreten. Statische Routings werden häufig von handelsüblichen Navigationssystemen ausgegeben.

\section{Dynamisches Routing}

Beim Dynamischen Routing wird bei der Berechnung die jeweils aktuelle Verkehrssituation als Kantenbewertung einbezogen. Dynamisch berechnete Routen stellen das theoretische Optimum zum angefragten Zeitpunkt dar. Der zugrundeliegende Straßengraph verändert sich kontinuierlich: Wegen sich ändernder Geschwindigkeiten oder Fahrzeiten erhöhen oder verringern sich die Kantenbewertungen. Die Qualität des Routingergebnisses ist jedoch stark abhängig von der Qualität und zeitlichen Korrektheit der verfügbaren Verkehrsinformationen. Dynamisches Routing ist vor allem in verkehrlich stark belasteten Gebieten zur Berechnung der schnellsten Route sinnvoll. Dynamisches Routing mit Berücksichtigung aller Verkehrsinformationen ist jedoch für Kraftfahrer derzeit noch nicht verfügbar.

\section{Intuitives Routing}

Unter Intuitivem Routing soll die Navigation und Wegfindung des Kraftfahrers auf Basis eigener Gewohnheiten, Erwartungen und Informationen (Erfahrungen, aktuelle Wahrnehmung, Verkehrsfunk etc.) verstanden werden. Der Kraftfahrer wählt vorausschauend auf Basis der wahrgenommenen Hinweise seine Route.

\section{Forschungsprojekt MINERVA}

Im Folgenden wird das Forschungsprojekt MINERVA (MINimierung der Staufolgen im Lieferverkehr in Ballungsräumen durch Effiziente Routingunterstützung im Fahrzeug und Verkehrsbildabhängiges Ankunftsavis), durchgeführt von der Forschungsgruppe Verkehrslogistik der Technischen Hochschule Wildau [FH], gefördert im Programm $\mathrm{FH}^{3}$ des Bundesministeriums für Bildung und Forschung, vorgestellt. 


\subsection{Zielstellung und These}

Die aktuelle Verkehrssituation in städtischen Gebieten ist auf Grund kurzfristig entstehender und unvorhersehbarer Verkehrsstörungen schwer vorherzusagen. Einerseits sind die für den Kraftfahrer verfügbaren Verkehrsinformationen (Radio, Tageszeitungen, TMC etc.) oft ungefiltert, unvollständig, veraltet und/oder zum Zeitpunkt der Entscheidungsfindung nicht verfügbar. Andererseits sind umfangreiche Verkehrsinformationen für Ballungsgebiete, beruhend auf Detektorsystemen, Baustellenprotokollen und Prognosealgorithmen, in Verkehrsmanagementzentralen vorhanden. Gegenstand des Forschungsprojektes MINERVA ist die Nutzbarmachung dieser Informationen durch die Kombination der verfügbaren Verkehrsinformationen mit der Erfahrung und Ortskenntnis des Kraftfahrers und anschließend die Analyse der Wirkung auf das Intuitive Routing von Kraftfahrern im Vergleich zu anderen Routingansätzen. Die Forschungsthese besagt, dass die Bereitstellung von on-board-Verkehrsinformationen die intuitive Routenwahl des ortskundigen Kraftfahrers verbessert und somit Zeit, Distanz und Kraftstoff eingespart werden können.

\subsection{Technischer Aufbau}

Im Forschungsprojekt MINERVA wurde eine Applikation entwickelt, die die Informationsübermittlung zwischen Verkehrsmanagementzentrale Berlin, dem mobilen Endgerät des Kraftfahrers und der jeweiligen Speditionszentrale sicherstellt (siehe Abbildung 2). Die jeweils aktuellen Verkehrsinformationen und die Verkehrslage werden in regelmäßigen, kurzen Abständen über eine Schnittstelle von der Verkehrsmanagementzentrale an das MINERVA-System und anschließend per GPRS an das mobile Endgerät übermittelt. Anhand der Tourenplanung und der jeweils aktuellen Position des Kraftfahrers ermittelt das mobile Endgerät die Verkehrsinformationen, die sich im Gebiet zwischen seiner aktuellen Position und dem nächsten Ziel befinden. Nur diese relevanten Verkehrsinformationen werden auf dem mobilen Endgerät angezeigt.

\subsection{Feldversuch}

Ein wesentlicher Teil des Forschungsprojekts MINERVA ist die Erprobung der Lösung in einem Feldversuch. In der Zeit von Januar 2008 bis Februar 2009 waren sieben Berufskraftfahrer (ReiCo Spedition $\mathrm{GmbH} \&$ Co. KG und Frischdienst Berlin) im Stadtgebiet Berlin und im Umland mit den mobilen Endgeräten unterwegs. Diese

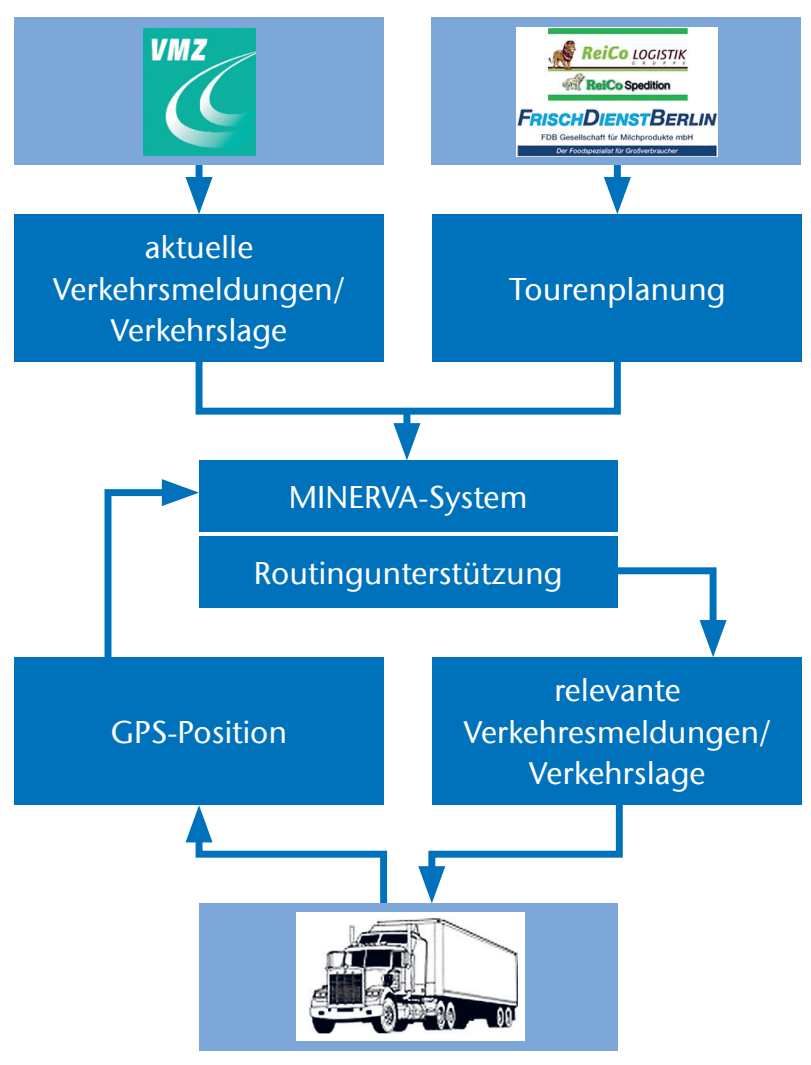

Abb. 2: Informationsübermittlung MINERVA

Kraftfahrer verfügen über langjährige Erfahrungen in der Auslieferung im Untersuchungsgebiet, sodass von einer sehr effizienten Fahrweise ausgegangen werden kann. Desto interessanter ist die Frage, ob durch die OnBoard-Bereitstellung von relevanten Verkehrsinformationen das Routing dieser erfahrenen Fahrer verbessert werden kann.

Der Feldversuch war in vier Phasen mit ansteigender Informationsunterstützung unterteilt, um festzustellen, wie das Intuitive Routing stufenweise besser unterstützt werden kann:

- Unterstützungsstufe 0: Aufzeichnung der Fahrtverläufe der Kraftfahrer ohne zusätzliche Verkehrsinformationen

- Unterstützungsstufe 1 »Pre-Trip-Verkehrsinformationen«: täglich ein Papierausdruck der am Vortag prognostizierten Verkehrslage als Karte und Verkehrsinformationen als Text

- Unterstützungsstufe 2 »Text-Information«: Textmeldungen der für das Tourengebiet relevanten Verkehrsinformationen auf mobilem Endgerät

- Unterstützungsstufe 3 »Digitalisiertes Verkehrslagebild «: GIS-Karte für das Tourengebiet, auf der die Straßen entsprechend der Verkehrslage gelb und rot eingefärbt sind, und Textmeldungen auf mobilem Endgerät 


\section{Erste Ergebnisse aus dem Forschungsprojekt MINERVA}

\subsection{Charakterisierung der Testgruppe} »am Feldversuch teilnehmende Kraftfahrer«

Am Feldversuch waren erfahrene Berufskraftfahrer der Lebensmittelauslieferung an Großkunden (zum Beispiel Hotels, Krankenhäuser, Einzelhändler) mit Fahrzeugen mit 12 und 18 t zGG (zulässiges Gesamtgewicht) beteiligt, die über langjährige Kenntnis ihres Tourengebiets verfügen. In der Regel sind die teilnehmenden Kraftfahrer im Auswertungszeitraum eine Tour pro Tag gefahren, nur zu 6,7 \% wurden zwei Touren pro Tag gefahren, dabei war die zweite Tour am Tag kürzer als die erste. Die durchschnittliche Anzahl der Stopps pro Tag betrug 17,4 und schwankt im Wochenverlauf, so sind vor allem Montag (Auffüllen der Vorräte) und Freitag (Bevorratung für das Wochenende) auslieferstarke Tage (siehe Abb. 3). Durchschnittlich ist jeder Kraftfahrer täglich $116 \mathrm{~km}$ vom Tourenbeginn bis zum letzten Stopp gefahren. Die Schwankungen der Fahrleistung im Wochenverlauf folgen der Anzahl der Stopps pro Tag (siehe Tabelle 1), ebenso die Verweildauer im öffentlichen Straßenraum (Fahrzeit plus sonstige Zeiten) von durchschnittlich 7 h 48 min pro Tag (siehe Abb. 4).

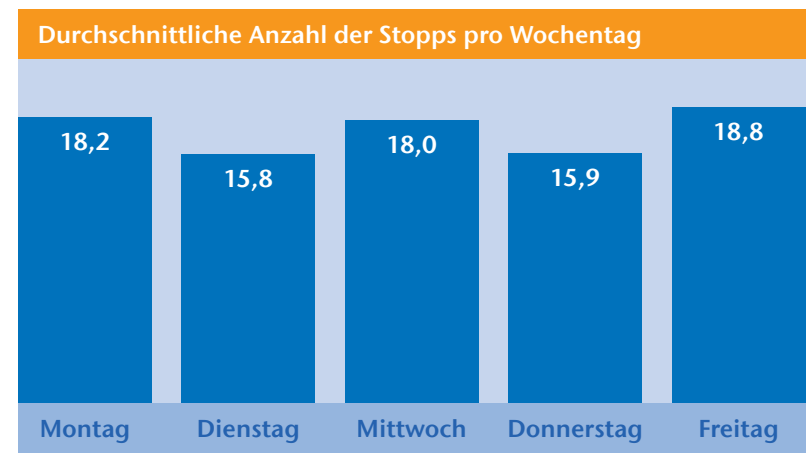

Abb. 3

\begin{tabular}{|l|l|l|l|}
\hline & Minimum & Maximum & Durchschnitt \\
\hline Montag & $23,5 \mathrm{~km}$ & $330,6 \mathrm{~km}$ & $127,2 \mathrm{~km}$ \\
\hline Dienstag & $23,5 \mathrm{~km}$ & $246,2 \mathrm{~km}$ & $106,3 \mathrm{~km}$ \\
\hline Mittwoch & $23,0 \mathrm{~km}$ & $243,0 \mathrm{~km}$ & $116,4 \mathrm{~km}$ \\
\hline Donnerstag & $20,2 \mathrm{~km}$ & $268,4 \mathrm{~km}$ & $108,7 \mathrm{~km}$ \\
\hline Freitag & $24,9 \mathrm{~km}$ & $264,0 \mathrm{~km}$ & $122,4 \mathrm{~km}$ \\
\hline
\end{tabular}

Tab. 1: Durchschnittliche Fahrleistung pro Wochentag

\subsection{Vergleich der Routingansätze}

Anhand einer Stichprobe aus den aufgenommenen Daten wurde ein Vergleich der verschiedenen Routingansätzen durchgeführt. Dabei war die Frage zu klären, wie

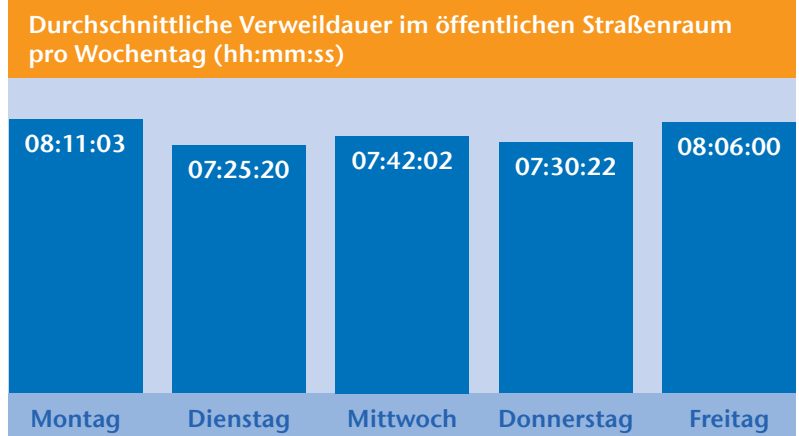

Abb. 4

und auf welchem Netz die Vergleiche durchzuführen sind. Mögliche Netze für den Vergleich sind in Tabelle 2 gegenübergestellt. Das Straßennetz mit realer Belastung SN3 wäre das am besten geeignete Netz für einen Vergleich, jedoch sind die damit verbundenen Anforderungen an Datenverfügbarkeit und Aktualität gegenwärtig nicht realisierbar.

\begin{tabular}{|l|l|l|}
\hline $\begin{array}{l}\text { Straßennetz ohne } \\
\text { Verkehrsinfor- } \\
\text { mationen (SN1) }\end{array}$ & $\begin{array}{l}\text { Straßennetz mit } \\
\text { Prognose- } \\
\text { belastung (SN2) }\end{array}$ & $\begin{array}{l}\text { Straßennetz mit } \\
\text { realer Belastung } \\
\text { (SN3) }\end{array}$ \\
\hline $\begin{array}{l}\text { Kantenbewertung: } \\
\text { Durchschnitts- oder } \\
\text { Höchstgeschwindig- } \\
\text { keiten }\end{array}$ & $\begin{array}{l}\text { Abbildung der } \\
\text { prognostizierten } \\
\text { Verkehrslage in drei } \\
\text { Abstufungen }\end{array}$ & $\begin{array}{l}\text { Reale Verkehrslage } \\
\text { auf den Straßen }\end{array}$ \\
\hline & $\begin{array}{l}\text { Berücksichtigung } \\
\text { aller bekannten } \\
\text { Verkehrsstörungen }\end{array}$ & $\begin{array}{l}\text { Oft sehr kurzfris- } \\
\text { tiges Auftreten/ } \\
\text { Verschwinden von } \\
\text { Verkehrsstörungen }\end{array}$ \\
\hline & $\begin{array}{l}\text { Aktualisierung der } \\
\text { Prognose alle 15 } \\
\text { min. }\end{array}$ & $\begin{array}{l}\text { Verkehrsbelastungen } \\
\text { haben sehr unter- } \\
\text { schiedlich starke }\end{array}$ \\
\hline
\end{tabular}

Tab. 2: Charakterisierung der unterschiedlichen Straßennetze

Unter Prognosebelastung (SN2) soll die Prognose der Verkehrsmanagementzentrale über die Verkehrssituation in den nächsten 15 min anhand der jeweils gültigen Verkehrsinformationen wie Stau, Baustellen etc. und der aus diesen Informationen und aus aktuellen Verkehrsmessungen resultierenden Berechnung der Verkehrsbelastung der einzelnen Kanten verstanden werden.

Um die Qualität des gewählten Straßennetzes SN2 gegenüber einem Straßennetz ohne aktuelle Verkehrsinformationen (SN1) zu ermitteln, wurden Test-Routings mit Touren aus der Stichprobe durchgeführt. Dazu wurden Start- und Zielpunkte der Touren durch 
eine statische Routengenerierung anhand eines Navigationssystems verbunden (statisches Routing) und die empfohlene Route auf dem Straßennetz mit Prognosebelastung (SN2) simuliert. Parallel wurden die Touren mit einem dynamischen Routing simuliert. Abb. 5 zeigt, dass auf einem Straßennetz mit Prognosebelastung (SN2) statische Routings deutlich schlechter abschneiden als dynamische Routings.

Simulationsergebnisse
57:36
21:36:24
14:24
76:00:12
00:00
Fahrzeit (mm:ss)

Abb. 5: Simulationsergebnisse des statischen und dynamischen Routings unter Berücksichtigung der im MINERVA-System verfügbaren Prognosebelastung

Deswegen wurde das Straßennetz mit Prognosebelastung (SN2) für den weiteren Vergleich zwischen intuitivem Routing mit und ohne MINERVA-Unterstützung herangezogen. Es wurden drei Routing-Varianten miteinander verglichen:

- dynamisches Routing - optimale (schnellste) Route auf SN2

- intuitives Routing mit MINERVA-Unterstützungsstufe 3 - vom Kraftfahrer im realen Straßennetz gefahrene Route

— intuitives Routing ohne MINERVA (Unterstützungsstufe 0) - die vom Kraftfahrer (zu einem anderen Zeitpunkt) üblicherweise gefahrene Route

Um die Repräsentativität zu sichern, wurden für den Vergleich oft gefahrene Routen der Fahrer aus dem Feldversuch ausgewählt. Für diese Routen wurden alle drei Varianten simuliert. Dabei wurden jeweils mit der Prognosebelastung (SN2) - zum Zeitpunkt der Fahrt mit MINERVA-Unterstützungsstufe 3 - alle Varianten simuliert. Für den Vergleich der verschiedenen Rou- tingansätze wird das dynamische Routing als Benchmark (100\%) verwendet, da es durch die Berücksichtigung der Verkehrsbelastung in SN2 das optimale Ergebnis liefert.

Die Auswertung des Feldversuches hat gezeigt, dass das intuitive Routing mit MINERVA im Straßenverkehr eine bessere Route ergibt, als das intuitive Routing ohne MINERVA-Unterstützung. Die Kraftfahrer haben durch die MINERVA-Unterstützung mit on-boad-Verkehrsinformationen den Vorteil, dass sie vor allem kurzfristig auftretende und unvorhersehbare Verkehrsstörungen großräumiger umfahren können und nicht erst, wenn sie die Verkehrsstörung vor sich sehen oder schon direkt betroffen sind.

Die Routingergebnisse auf einem Straßennetz mit Prognosebelastung (SN2) sind mit den Routings der Kraftfahrer auf einem realen Straßennetz (repräsentiert durch Straßennetz mit realer Belastung SN3) quantitativ nicht vergleichbar. Unterschiede zwischen beiden Netzen ergeben sich einerseits, weil die reale Verkehrsbelastung aller Kanten im realen Straßennetz gegenwärtig und in absehbarer Zeit nicht ermittelt werden kann. So ist zum Beispiel das Straßennetz nicht vollständig mit Detektorsystemen zur Aufnahme der aktuellen Verkehrssituation ausgestattet. Andererseits treten Verzögerungen zwischen Entstehung und Integration der Verkehrsinformationen auf, sodass reale Verkehrsinformationen bei der Übernahme in das Straßennetz mit Prognosebelastung (SN2) schon veraltet sein können.

\section{Schlussfolgerungen und Ausblick}

Der Vergleich der Routingansätze zeigt, dass sich die intuitive Routenwahl erfahrener Berufskraftfahrer durch die On-Board-Verkehrsinformation mit dem MINERVASystem verbessern lässt, da eine Kombination aus intuitivem Routing und relevanten Verkehrsinformationen eine sehr gute Entscheidungsgrundlage bildet: So hat der Kraftfahrer die Möglichkeit, über seinen Sichtradius hinaus, entsprechend der Verkehrslage zu reagieren. In der Stichprobe haben die Kraftfahrer mit MINERVA eine Verkürzung der Fahrzeit von 5 \% gegenüber dem intuitiven Routing erreicht. Für weniger erfahrene Kraftfahrer dürfte das Optimierungspotenzial noch deutlich höher liegen, da die Verbesserung bei den erfahrenen Berufskraftfahrern als ein Minimalwert angesehen werden kann. 
Hinsichtlich der ökologischen Wirkungen des MINERVA-Systems wurde abgeschätzt, dass sich pro Lkw ca. $715 \mathrm{~kg} \mathrm{CO}$ einsparen ließen (bei durchschnittlich $22.280 \mathrm{~km} / \mathrm{Jahr}, 3 \% \mathrm{~km}$-Einsparung durch MINERVA, angenommenen $5 \mathrm{t}$ durchschnittlicher Nutzlast, 0,214 $\left.\mathrm{g} \mathrm{CO}_{2} / \mathrm{tkm}\right)$.

Das dynamische Routing liefert aus mathematischer Sicht das beste Ergebnis, es ist jedoch sehr stark abhängig vom Umfang und der Qualität der zugrundeliegenden Verkehrsinformationen. Da zurzeit keine vollständig realitätstreue Abbildung der Verkehrssituation in städtischen Gebieten verfügbar und möglich ist, ist das dynamische Routing für die praktische Anwendung ein suboptimales Ergebnis. Um ein vollständiges Netz mit realen Belastungswerten zu erstellen, könnten zum Beispiel Floating Car Data mit einer sehr großen Flotte verwendet werden. In diesem Bereich besteht noch Forschungsbedarf.

Das MINERVA-System kann sowohl von Speditionskraftfahrern angewendet werden, die sich im Stadtgebiet sehr gut auskennen, da es keine Route vorgibt, sondern »nur« Verkehrsinformationen zu Verfügung stellt. Hohen Nutzen hat es vor allem für Kraftfahrer, deren Touren nicht jeden Tag identisch sind. Weiterhin ist das MINERVA-System auf Taxi- und Kurierdienste übertragbar. Auch Privat- und Geschäftsleute, die viel im Stadtgebiet unterwegs sind und zeitkritische Fahrten durchführen, können vom MINERVA-System profitieren.

Eine Übertragung des MINERVA-Systems in andere Ballungsgebiete ist möglich, Vorraussetzung ist die Verfügbarkeit von umfangreichen und aktuellen Verkehrsinformationen über eine Schnittstelle. Um Verkehrsinformationen über das MINERVA-System jedoch gebietsübergreifend zur Verfügung stellen zu können, ist - über TMC (Traffic Message Channel) hinaus - ein Standard für das Format von Verkehrsinformationen und die Zusammenarbeit der verschiedenen Anbieter von Verkehrsinformationen in den Regionen notwendig.

\section{Literatur}

Dasen, Stefan; Janisch, Katrin; Meimbresse, Bertram; Rommerskirchen, Stefan; Schad, Helmut (2001): Piloterhebung zum Dienstleistungsverkehr und zum Gütertransport mit Personenwagen - Bericht zum Forschungsauftrag SVI 1999 / 327 (36 / 00) im Auftrag des Bundesamtes für Straßen (Schweiz).

Dijkstra, Edsger Wybe (1959): A note on two problems in connexion with graphs. Numeric Mathematics I, S. 269 ff.

Hart, Peter; Nilsson, Nils; Raphael, Bertram (1968): A formal basis for the heuristic determination of minimum cost paths. IEEE Transactions on Systems Science and Cybernetics SSC4 (2), S. 100-107.

Schultes, Dominik (2008): Route planning road networks. Dissertation, Universität Fridericiana zu Karlsruhe (TH).

Wermuth, Manfred; Binnenbruck, Horst H.; Machledt-Michael, Sonja; Rommerskirchen, Stefan; Sonntag, Herbert; Wirth, Rainer (1998): Bestandsaufnahme notwendiger und verfügbarer Daten zum Wirtschaftsverkehr als Grundlage pragmatischer Datenergänzungen - Schlussbericht zum Forschungsprojekt FE 01.145 G96C, im Auftrag des Bundesministeriums für Verkehr.

\section{Autoren}

Dipl.-Ing. Bertram Meimbresse

TH Wildau [FH], Forschungsgruppe Verkehrslogistik Tel. +49 3375 508-355, bertram.meimbresse@tfh-wildau.de

Dipl.-Wirtsch.-Ing. (FH) Christine Behnke TH Wildau [FH], Forschungsgruppe Verkehrslogistik Tel. +49 3375 508-129, christine.behnke@tfh-wildau.de

Prof. Dr.-Ing. Herbert Sonntag

TH Wildau [FH], Forschungsgruppe Verkehrslogistik Tel. +493375 508-924, herbert.sonntag@tfh-wildau.de 